\title{
OHNE PANZERHEMD DER GEWOHNHEITEN
}

\author{
Über das Wahrnehmen der Stimme und den Stellenwert \\ von Spracherfahrungen im Fachbereich ,Deutsch als \\ Zweitsprache' 1
}

\section{Simone Hein-Khatib}

.1 Der Ausdruck ,Panzerhemd der Gewohnheiten' ist dem Gedicht ,Vypálenci' (,Ausgebrannte') des tschechischen Dichters Jan Skácel in der deutschen Übersetzung von Reiner Kunze entnommen. In: Kunze 2003: .188

Der Essay versucht, sich einer Dimension unserer Sprachlichkeit zu nähern, die im Gemeinsamen Europäischen Referenzrahmen für Sprachen oder in einschlägigen Diagnostikverfahren zur Bestimmung der Sprachkompetenz keine Beachtung findet: der Wahrnehmung der Stimme. Die Verfasserin wählt hierfür den Weg der Introspektion. Durch den Einbezug von Notizbucheintragungen und die Reflexion eigener Hör-Erfahrungen in der Erstsprache Deutsch und in den zwei später gelernten Sprachen Arabisch und Tschechisch geht sie der Frage nach, inwiefern ihr Zuhören und ihr Wahrnehmen der Stimme von ihrer Sprachkompetenz in der jeweiligen Sprache geprägt werden. Im Nachwort wird der Entstehungshintergrund des Essays - der Stellenwert von Spracherfahrungen im Fachbereich ,Deutsch als Zweitsprache' - näher beleuchtet.

Während ich über Stimmen und ihre Wirkung auf mich nachdenke, fällt mir auf, dass ich mich vor allem an Stimmen erinnere, die Arabisch oder Tschechisch sprechen.

„Der Oktober neigt sich dem Ende zu. Es ist Freitag, am frühen Abend. Pavel und ich sind auf dem Weg von Sušice nach Ústí nad Labem - knapp $250 \mathrm{~km}$ vom Fuße des Böhmerwaldes in den Nordwesten Tschechiens. Die Hügel, Wälder und Sträucherreihen, die am Autofenster vorbeiziehen, sind in der Abenddämmerung nur mehr schemenhaft zu erkennen. Meine Gedanken hängen im Karussell des Ungelösten und Unerledigten fest. Pavel stellt das Navigationsgerät an, das er vor kurzem von seinem Vater geschenkt bekommen hat. Er spielt mir einige Stimmen vor, weibliche und männliche, manche sprechen Tschechisch, andere klingen, als ob Deutschsprachige tschechische Wörter ablesen, ohne je zuvor mit der tschechischen Lautung in Berührung gekommen zu sein. Bei keiner der Stimmen kann ich 
mir ein menschliches Gesicht dazu vorstellen. Am unerträglichsten sind die monotonen Computerstimmen. Dann erklingt plötzlich eine Stimme, die nichts mit den vorherigen gemein hat. Sie spricht das gleiche begrenzte Repertoire an Einzelwörtern und Wendungen wie die Stimmen zuvor: „doprava“ (nach rechts), „za tři sta metrů sjed'te“ (fahren Sie nach dreihundert Metern ab), „ted' sjed'te“ (fahren Sie jetzt $a b$ ) ..., und doch krempelt diese Stimme alles um. Es ist, als würde den wenigen, immer gleichen Worten plötzlich Leben eingehaucht, Leben in all seinen Schattierungen und Unebenheiten, in seiner Brüchigkeit, Flüchtigkeit und Zartheit, seiner Unvollkommenheit und seiner zuweilen schrägen Komik. Diese Stimme hat ein Gesicht, es ist das Gesicht von Pavel Liška, einem tschechischen Schauspieler. Seine Stimme festzumachen, zu analysieren, wie er spricht - absolut unmöglich, ja vermessen, so als wolle man das, was das Lebendigsein ausmacht, in eine Definition pressen. Das Gedankenkarussell in meinem Kopf hat sich abgeschaltet, jetzt sitze ich hier im dunkelblauen Opel auf dem Beifahrersitz, fahre mit Pavel durch die Nacht dieses Oktoberfreitags, betrachte die Lichter der entgegenkommenden Autos und die schattenhaften Fetzen der Umgebung, die für einen Augenblick aus der Dunkelheit aufflackern. Dem Wochenende entgegen. "(Notizbuch, 2009)

„Ich bin von der Uni zu Fuß zum Hotel gegangen, durch nasse weiße Flocken, die sich auf den beleuchteten Straßen in grauschwarzen Schneematsch verwandelten. Im schmalen Hotelzimmer angekommen, setze ich mich auf das Bett, stelle mein Laptop auf den Stuhl und schiebe eine DVD ein.

Die vergangenen drei Jahre waren Jahre des Umbruchs. Ich bin in ein neues Land und eine neue Sprache gezogen, habe zudem mit über vierzig nochmals einen neuen Beruf erlernt. In keinem der drei bin ich angekommen, komme mir vor wie disparates Stückwerk. Im Tschechischen stocke ich, und ich bange, das Arabische zu verlieren. Seit wir in Sušice wohnen, habe ich nur selten mit jemandem auf Arabisch gesprochen. Irgendwann begann ich, nach arabischsprachigen Filmen Ausschau zu halten. Der Film, den ich an diesem Abend bei mir habe, ist ein Dokumentarfilm über jüdische Israelis irakischer Herkunft, gedreht vom in der Schweiz lebenden Regisseur Samir, selbst Sohn eines irakischen Vaters und einer Schweizer Mutter. ${ }^{2}$ Samir führt im Film längere Gespräche mit fünf Personen, unter ihnen die Schriftsteller Shimon Ballas und Sami Michael, die in den 1950er Jahre aus dem Irak nach Israel migrierten. Obwohl sie irakisches Arabisch sprechen und ich vor allem das palästinensische Arabisch gewohnt bin, verstehe ich sie gut. Und nicht nur die Sprache, die ganze Art, wie Shimon Ballas und Sami Michael von ihren Erfahrungen erzählen, ist mir sehr vertraut. Je länger ich ihnen zuhöre, umso stärker überkommt mich ein eigenartiges Gefühl, das ich nur unzureichend zu beschreiben vermag. Eine Art von ,Zuhause-' und zugleich ,Im-Leben-Sein', ähnlich wie wenn man vom Verkehrslärm einer mehrspurigen Straße in einen Konzertsaal tritt und plötzlich umgeben ist vom Klang der Musik. Dass dies allein durch die selten gehörte arabische Sprache ausgelöst wird, ist unwahrscheinlich. Sprachen wirken immer über die Stimmen der Menschen, die sie sprechen. Im Fall von Shimon Ballas und Sami Michael sind es weitsichtige und lebenskluge Stimmen, die

\footnotetext{
${ }^{2}$ Der Film trägt den Titel ,Forget Bagdad إنْسَ بغداد שכח מבגדד - jews and arabs - the iraqi connection.'
} 
die ideologischen Schubladen von ,den Arabern' und ,den Juden' nach einem Satz zerbröckeln lassen. " (Notizbuch, 2011)

„Freunde aus Deutschland waren zu Besuch. Den ganzen Tag sind wir durch den zaghaft beginnenden Frühling in den Hügeln und Wäldern Šumavas gewandert. Am Abend waren alle angenehm erschöpft und unsere Gäste wollten gerne einen tschechischen Film ansehen, auf Deutsch. Wir haben einige tschechische Filme in deutscher Synchronisation und ließen sie auswählen. Die Entscheidung fiel für ,Vratné láhve'3, einen heiter-bitteren Film über die Schwierigkeiten, sich mit dem Älterwerden zu versöhnen. Zdenëk Svĕrák hat das Drehbuch für den Film geschrieben und spielt selbst die Hauptrolle darin. In Tschechien ist Zdeněk Svĕrák als Drehbuchautor, Dramaturg und Schauspieler eine Berühmtheit, eine seltene Verbindung von Widerständigkeit und Leichtigkeit. Ich hatte ihn zuvor schon in Theaterstücken und Filmen auf Tschechisch gesehen, auch im Film,Vratné láhve', nun also das erste Mal auf Deutsch.

Es war schwer auszuhalten! Ich musste mit mir kämpfen, um bis zum Ende des Films auszuharren und nicht zuvor das Zimmer zu verlassen. Das, was fast schmerzlich irritierte, hatte weniger mit dem Sprachwechsel vom Tschechischen ins Deutsche zu tun, vielmehr betraf es den Klang, den Klang von Zdenĕk Svĕrák. Es war Un-Stimmig-keit im wörtlichen Sinne. Jedes Mal, wenn Zdenĕk Svĕrák etwas sagte, erschrak ich erneut darüber, wie die Einheit seiner Person, die Einheit von Stimme und Körper, vor meinen Augen zerbrach. Zdenĕk Svĕrák war existent als sichtbarer Körper, aber dieser war mit einem Klang ausgestattet, der in keinster Weise zu ihm gehörte. Als der Film zu Ende war, spürte ich, wie mein Körper aufatmete, endlich entlassen aus der Zerreißprobe." (Notizbuch, 2012)

„Der protestantische Friedhof in Haifa, früh am Morgen. In der Dämmerung gehören die Geräusche noch ganz der Natur. Einzelne Vogelstimmen sind zu hören, leises Rascheln von Blättern im Wind. Mohammed Bakri ${ }^{4}$ steht am Grab von Emil Habibi, ins Gespräch mit dem verstorbenen Freund vertieft. ${ }^{5}$ Er spricht zu ihm über gemeinsam Erlebtes, dann über Ereignisse, die sich seit dem Tod des Freundes zugetragen haben, Ereignisse, die die Palästinenser im Land und auch Mohammad Bakri und seine Familie unmittelbar erschütterten. Ereignisse voller Gewalt, Zerstörung, Verzweiflung und Verleumdung.

Von Mohammad Bakris Stimme trage ich einen deutlichen Klangeindruck in meinem Gedächtnis. Regelmäßig schaue (höre) ich mir seine Interviews an, die er mit unterschiedlichen Menschen über ihre Lebenserfahrungen als Palästinenser in Israel führt ${ }^{6}$. Rufe ich mir jedoch seine Stimme in Erinnerung, so ist sie stets ver-

\footnotetext{
${ }^{3}$ Auf Deutsch läuft der Film unter dem Titel ,Leergut' (Regie: Jan Svěrák).

${ }^{4}$ Mohammad Bakri ist ein in Israel lebender palästinensischer Schauspieler und Regisseur.

${ }^{5}$ Emil Habibi $(1922$ - 1996) war ein palästinensischer Schriftsteller und Journalist. Mohammad Bakri hat Emil Habibis berühmten Roman ,Der Peptimist' bereits zu dessen Lebzeiten international als Ein-Mann-Theaterstück aufgeführt. Mehrmals sind die beiden zu diesen An-lässen gemeinsam ins Ausland gereist.

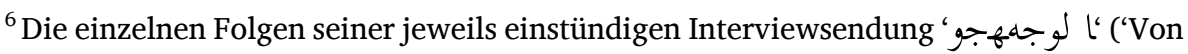
Angesicht zu Angesicht') sind im online-Archiv des palästinensischen Fernsehens (Palestine Bublic Broadcasting Corporation) zu sehen unter: http : / / ww . pbc . ps /Programs $/ \%$ D9\%88\%
} 
bunden mit dieser Szene in seinem Dokumentarfilm, Since you left'. Im Film ist der Haifaer Friedhof ein Ort des Kontrasts, einer der wenigen Orte, wo das aggressive Trommelfeuer im Land aussetzt. Ein Ort der Auszeit, an dem Mohammad Bakri für sich ist. Der Kontrast zu den anderen Szenen des Filmes ist besonders akustisch wahrnehmbar. Vor der zarten Klangkulisse der Naturgeräusche ist das Ohr ganz auf Mohammad Bakris ruhiges Sprechen konzentriert, auf sein Gespräch mit dem verstorbenen Freund. Ein intimes Gespräch, intim nicht aufgrund vermeintlicher persönlicher Offenbarungen, intim v or a llem durch die S timme und die Sprechweise: als habe ein Mensch unmittelbar neben einem Platz genommen, als lege er seine Schutzschilder ab und beginne zu sprechen, ließe seinen Erinnerungen, seinen Gedanken und Gefühlen freien Lauf.

Selten rückt im Alltag das Leben unbekannter Menschen in solcher Dichte und Nähe an uns heran. Selten auch geschieht es im Alltag, dass wir einem Menschen zuhören und dabei bewusst auf seine Stimme achten. In Gesprächen läuft der Klang der Stimme meist unbemerkt nebenher, unser Bewusstsein ist ganz auf das Erfassen des Inhalts ausgerichtet. Unser Gegenüber erwartet eine Antwort auf das, was er sagt; wir können uns nicht dem Zuhören hingeben, geschweige denn den Klang seiner Stimme und ihre Nachwirkungen auf uns aufnehmen. Genau dies aber ist bei Filmen möglich: ein Zuhören ohne die Anforderung, sogleich reagieren zu müssen. Filme erlauben uns, offen z u w erden für d en K lang v on Stimmen. "(Notizbuch, 2015)

Woran mag es liegen, dass es vor allem Stimmen im Arabischen und Tschechischen sind, an die ich mich erinnere, nicht aber im Deutschen?

Ich hege den Verdacht, dass ich Stimmen im Tschechischen und Arabischen anders wahrnehme als im Deutschen, und dass dieses ,anders Wahrnehmen' damit zu tun hat, wie sich die Sprachen in mir eingewurzelt haben, in welcher Weite und Tiefe mir das Bedeutungsspektrum ihrer Wrter und grammatischen Strukturen vertraut ist.

Mein Arabisch und mein Tschechisch haben keine Kindheit. Ich war achtzehn, als ich der arabischen Sprache das erste Mal in Israel-Palästina begegnete. Richtig gelernt habe ich sie nach meiner Rückkehr nach Deutschland, sozusagen exterritorial. Vor allem von meinem ersten Mann, einem Palästinenser aus Israel, der mit mir nach Deutschland zog. Außer einem Jahr in Israel-Palästina habe ich nie für längere Zeit in einem arabischsprachigen Land gelebt und war dennoch siebzehn Jahre lang im privaten Leben nahezu täglich vom Arabischen umgeben. Als ich Tschechisch zu lernen begann, war ich schon Mitte dreißig. Die ersten Jahre habe ich es ebenfalls ,exterritorial' in Deutschland gelernt, von dem Mann, der heute mein Lebensgefährte ist, und aus Büchern. Von 2008 bis 2013 haben wir in Tschechien gelebt, von wo aus ich nach Deutschland zur Arbeit pendelte. Arabisch und Tschechisch sind Sprachen, die wichtige Bereiche meines Lebens nach der Kindheit geprägt haben und prägen: sie sind Sprachen der privaten Orte und der persnlichen Beziehungen, mit einer eingeschränkten öffentlichen und ohne eine berufliche 
Sphäre. Vom Englischen, das ich in der Schule gelernt habe und in dem ich fast täglich lese, unterscheiden sie sich durch ihren Lebensbezug: ich habe nie längere Zeit in einem englischsprachigen Land oder mit einer englischsprachigen Person zusammen gelebt. Englisch ist für mich eine Lesesprache, eine Augensprache. Arabisch und Tschechisch, in denen ich auch lese, sind hingegen in erster Linie Ohrensprachen, Sprachen des persnlichen Gesprächs, Stimmensprachen. Bevor ich begann, Arabisch und Tschechisch zu lernen, hrte ich den spezifischen Klang dieser Sprachen. Seitdem ihre Laute Bedeutungen tragen, vermag ich das nicht mehr - wie auch im Deutschen nicht. Seitdem tragen diese Sprachen den stimmlichen Klang des Menschen, der sie spricht.

Die deutsche Sprache war - in zwei ihrer Ausprägungen: Schwäbisch und Hochdeutsch - in meinem Leben von Anfang an da. Sie war die Sprache, in der ich vom ersten Moment an angesprochen und ins Leben eingeführt wurde. In ihr habe ich die Welt um mich herum zu benennen gelernt, Lesen und Schreiben gelernt, meine ganze schulische und einen großen Teil meiner universitären Ausbildung erhalten.

Hre ich Menschen auf Deutsch sprechen, so sind ihre Worte angefüllt mit Informationen, die weit über den eigentlichen Inhalt der Worte hinausreichen. So hre ich, ob ein Wort gängig oder ungewhnlich ist, ob es zum Korsett eines Klischees gehrt oder Konventionen sprengt. Ich hre, ob Worte etwas vage bezeichnen oder punktgenau treffen, ob sie einen Sachverhalt erhellen oder eher im Dunklen belassen. Ich spüre, ob Worte dem Thema, der Situation und dem Gesprächspartner angemessen sind, sie verfehlen oder sie gar missachten. Die Stimme einer Person teilt mir nicht nur ihr Alter und Geschlecht mit, vielmehr hre ich aus der Melodie, dem Rhythmus und der Klangfarbe der Laute auch die Region heraus, in der sie aufgewachsen ist, und ich erahne die gesellschaftliche Schicht, deren Sprechweise sie sich - bewusst oder unbewusst - zu Eigen gemacht hat. Und selbst dann, wenn mein Gegenüber sein Innenleben zu verbergen sucht, offenbaren mir seine Stimme und die Art, wie er spricht, seine gegenwärtige Stimmung und das ihm eigene Temperament. Ich hre seine Angespanntheit oder Freude, ob er gelster Stimmung ist, nervs, ruhig, erschpft, wütend oder traurig. ${ }^{7}$ Jedes Mal, wenn ich jemandem zuhre, der etwas auf Deutsch sagt, vernehme ich - meist ohne bewusst darauf zu achten - gleichzeitig mit dem eigentlichen Inhalt seiner Worte diesen polyfonen Chor an Hinweisen zu seiner Person.

Und was hre ich, wenn jemand Arabisch oder Tschechisch spricht, Sprachen, die ich nur begrenzt beherrsche? Was klingt da für meine Ohren durch die Stimme einer Person hindurch?

\footnotetext{
${ }^{7}$ Der Begriff, Person' i st h ier b ezeichnend. E r l eitet sich v om l ateinischen, persona' ab, das sich zusammensetzt aus ,per' (durch, hindurch) und ,sonare' (klingen, tnen) bzw. ,sonus' (Klang, Ton, Laut). Man knnte es so deuten: eine Person gibt sich durch ihren Klang, durch ihr Sprechen und ihre Stimme zu erkennen.
} 
Wie im Deutschen identifiziert mein Gehr in arabischen und tschechischen Lautstrmen Worte und grammatische Strukturen; jedoch ist mein Verständnis dieser Worte stärker abhängig vom Themenbereich. Je fachspezifischer die Inhalte, umso mehr schrumpft das, was ich verstehe. Schimpft ein Automechaniker über einen beschädigten Kolben und erläutert mir dann im Detail die daraus entstehenden Beeinträchtigungen im Funktionieren des Motors, so ist das Vorstellungsbild, das dazu in meinem Kopf entsteht, weitaus nebulser, als wenn er mir von seiner vergesslichen Mutter erzählt. Aber nicht nur hinsichtlich der Spannweite meiner Wortschatzkenntnisse, auch im Verständnis der Worte selbst gibt es deutliche Unterscheide zwischen dem Deutschen und den zwei später gelernten Sprachen. So tue ich mir in letzteren schwer, das soziale Register eines Wortes einzuschätzen, ich bin unbeholfen im Entziffern d er G rade a n A lltäglichkeit o der G ehobenheit, a n Privatheit und Frmlichkeit. Meine Vorstellung der innersprachlichen Normalität ist im Arabischen wie im Tschechischen lückenhaft. Die Grenzlinien zwischen dem blichen und Außergewhnlichen erkenne ich undeutlich und den Spielraum für sprachliche Innovationen vermag ich nicht abzuschätzen. Auch über die Geschichte und Wertungen eines Wortes in der Gesellschaft weiß ich wenig. Meine Einschätzung der Präzision eines Wortes zum Bezeichnen eines Sachverhalts hat immer Unschärfen - ich überblicke weder das ganze zur Verfügung stehende Wrterspektrum, noch bin ich vertraut mit den feinen Bedeutungsunterschieden zwischen den Synonymen. Was ich im Arabischen und Tschechischen verstehe, ist der eigentliche Inhalt, die ,pure' Bedeutung der Worte, die Denotate, während mir viele der darüber hinausweisenden Bezüge entgehen.

Wie bei den Worten so gelingt es mir auch im stimmlichen Klang nur ungenau, die gesellschaftlichen Prägungen und Färbungen zu erkennen und die soziale und geographische Herkunft einer Person auszumachen. Weitaus hellhriger als im Deutschen nehme ich jedoch die Sorgfalt beim Aussprechen der einzelnen Laute wahr. Hre ich jemanden Arabisch oder Tschechisch sprechen, so registriere ich sogleich, ob er über die Laute hinweghuscht, sie verschluckt oder ihnen Nachdruck verleiht. Ebenso, ob jemand seine Sätze zu Ende führt oder dazu neigt, sie vorzeitig abzubrechen. Eindringlicher als im Deutschen nehme ich auch die Geschwindigkeit und den Rhythmus auf, das Fließen und Stocken, das Zusammenspiel zwischen Atmen und Sprechen, wie jemand Pausen und Betonungen setzt, ob der Atem die Sätze bis zu ihrem Ende trägt oder bereits zuvor ausgeht. Und ich achte bewusster auf die Bestimmtheit oder Zurückhaltung, die Festigkeit oder Kraftlosigkeit der Stimme, auf ihre Lautstärke und auf das Spektrum ihrer Variation, den Wechsel und die Spannweite zwischen ihren Hhen und Tiefen. All dies teilt mir Wichtiges über die sprechende Person mit, was ich aus ihren Worten allein nicht herauszuhren vermag.

Hre ich jemanden Arabisch oder Tschechisch sprechen, dann ist das, was ich seinem Sprechen entnehme, im Vergleich zum Deutschen auf eine spezifische Weise reduziert: ich höre den blanken Inhalt der Wörter ohne oder mit 
nur einer dünnen Stoffschicht eines gesellschaftlichen Gewandes; und ich hre aus der Stimme und Sprechweise die emotionale Verfassung des Sprechers heraus.

Liegt es an meinem stärkeren Angewiesensein auf den Einbezug des stimmlichen Klangs zur Interpretation der Worte, dass ich mich eher im Arabischen und Tschechischen an Stimmen erinnere als im Deutschen? Oder ist es das ,Weniger' an Informationsgehalt, das die Einheit aus Worten und Stimme in diesen Sprachen eindringlicher wirken lässt? hnlich wie einem SchwarzWeiß-Fotografien die Gesichtszüge eines Menschen oder bestimmte Strukturen von Pflanzen o der L andschaften oftmals e indrücklicher z eigen a ls vergleichbare Farbfotografien?

Das Zuhren geschieht in meinen später gelernten Sprachen nicht so beiläufig wie im Deutschen. Will ich verstehen, was jemand sagt, muss ich meine Ohren spitzen in einem Maße, wie ich es im Deutschen tue, wenn mich etwas sehr interessiert und ich keine Nuance verpassen mchte. Dieses anhaltende fokussierte ,ganz Ohr sein' kostet Kraft, so dass ich früher Zeichen von Erschpfung spüre als im Deutschen. Das Verstehen an sich - der Prozess, bei dem sich eine Vorstellung von dem, was ich vernommen habe, in meinem Kopf herausbildet - vollzieht sich im Arabischen und Tschechischen langsamer. Manchmal scheinen mir die Vorstellungsbilder schwächer zu sein, insbesondere dann, wenn sie nicht genug Zeit haben, sich zu entwickeln und in mir zu wirken - weil ich zu einer Reaktion aufgefordert bin oder bereits neue Sätze zu einem anderen Thema aufnehmen muss. Zuweilen fordere ich mich bewusst zur Konzentration auf, weil ich merke, dass die Sätze sonst an mir vorbei gleiten, ohne dass Vorstellungsbilder entstehen.

Wenn ich unter Menschen bin, beispielsweise im Zug oder einem Caf sitze, dann gelingt es mir leichter, den Inhalt der um mich herum geführten Gespräche in Tschechisch und Arabisch auszublenden, ihn als puren Stimmenklang wahrzunehmen und meinen eigenen Gedanken nachzuhängen, als wenn die Menschen um mich herum auf Deutsch sprechen. So, als gäbe es in den später gelernten Sprachen einen Schalter, der sich umlegen ließe von ,Inhalt-und-Klang-von-Stimmen' auf ,Nur-Klang-von-Stimmen'. Im Deutschen bin ich weitaus stärker in einem Automatismus der Inhaltsentschlüsselung um mich herumschwirrender Worte verfangen. Meist vermag ich mich nur dann aus ihm zu lsen, wenn meine inhaltliche Aufmerksamkeit anderweitig - z.B. durch ein Gespräch, ein unerwartetes Ereignis, einen spannenden Text - gefordert ist, nicht aber durch eine Wahrnehmungsverschiebung auf puren, inhaltslosen Stimmenklang. Zugleich ist mein Zuhren im Arabischen und Tschechischen leichter irritierbar durch akustische Strungen wie Verkehrslärm, laute Musik oder Gespräche am Nebentisch. Als wären die Bahnen, in denen das Begreifen der vernommenen Worte erfolgt, weniger tief in mir eingraviert und die Worte knnten leichter von anderen Klängen herauskatapultiert werden, bevor ich ihren Sinn erfasst habe. Neben der klanglichen 
Störanfälligkeit ist mein Zuhören in diesen beiden Sprachen auch stärker inhaltlichen Irritationen ausgesetzt. Unvergleichlich öfter als im Deutschen stößt es auf Wörter, die es nicht oder nur teilweise versteht. Dann setzt ein bewusster Prozess der Sinnsuche ein. Der Fokus verschiebt sich nach außen auf die Form des Wortes und auf den Kontext. Die klangliche Form des unbekannten Wortes wird nach Ähnlichkeiten mit schon Bekanntem abgetastet und die Passgenauigkeit von Bedeutungsmöglichkeiten im Kontext ausprobiert. Manchmal, wenn nötig und möglich, bitte ich mein Gegenüber auch direkt um eine Erklärung des unverstandenen Wortes. Ein Nebeneffekt davon ist, dass ich im Arabischen und Tschechischen eine weitaus vorsichtigere Zuhörerin bin. Höre ich im Deutschen jemandem zu, dann erfolgt ohne mein bewusstes Zutun in Bruchteilen einer Sekunde eine Grundbewertung zwischen angenehm/unangenehm, glaubwürdig/unglaubwürdig, interessant/uninteressant. Im Arabischen und Tschechischen halte ich die Schublade der Schlüsse und Urteile wesentlich länger offen und schließe sie auch mit geringerer Bestimmtheit: ich bin mir der Begrenztheit meines Verständnisses nur zu bewusst, weiß um die Leichtigkeit, mit der ich in meinem Verstehen daneben liegen könnte.

Zuhören in meinen zwei später gelernten Sprachen fordert von mir im Vergleich zum Zuhören im Deutschen ein Mehr an bewusster Konzentration. Es ist störanfälliger, langsamer, kräftezehrender und die Verbindung von stimmlichem Klang und Inhalt ist leichter aufkündbar; es ist offener und vorsichtiger, die eigene Fehlbarkeit immer einkalkulierend.

Sind Sehen und Zuhören vergleichbar? Meine Erfahrungen beim Zuhören in den drei Sprachen lassen mich an die Fotografen Josef Koudelka, Sebastião Salgado und Pennti Sammallahti denken, die ihr ganzes Leben immer wieder aufgebrochen sind, um zu fotografieren, oft in entlegene Gegenden.

Josef Koudelka, der 2013 in einem Interview sagt, dass er seit 43 Jahren nie länger als drei Monate in einem Land gelebt hat ${ }^{8}$, erzählt: „Einmal freundete ich mich mit einem jugoslawischen Rom an, einem tollen Kerl. Er sagte: ,Josef, du bist so viele Jahre gereist, hast nicht angehalten, hast so viele Menschen, Länder und Orte gesehen. Sag mir, wo ist es am besten? Wo würdest Du gerne bleiben wollen?' Ich schwieg. Als sich unser Zusammensein dem Ende näherte, fragte er erneut. Ich wollte nicht antworten, aber er drängte. Schließlich sagte er: ,Weißt du, ich habe es verstanden. Du willst nicht antworten, weil Du solch einen Ort noch nicht gefunden hast. Du reist, weil du diesen Ort immer noch suchst.' Ich antwortete ihm: ,Mein Freund, du liegst ganz falsch. Ich bemühe mich verzweifelt, diesen Ort nicht zu finden.'“9

Warum diese beharrlichen Ortswechsel, fragt man sich, warum diese unentwegten Aufbrüche ins Unbekannte? Die Antworten der Fotografen darauf

\footnotetext{
${ }^{8}$ Koudelka 2013, im Interview auf dem ,Look3 photography festival' in Charlottesville, Virginia am .2013.6.15

${ }^{9}$ Koudelka 2002: 144, Übersetzung aus dem Tschechischen von der Verfasserin.
} 
machen deutlich, dass für sie die Notwendigkeit, das Gewohnte zu verlassen, mit dem Sehen zu tun hat, genauer: mit einem bestimmten Geistes- und Sinneszustand, den sie für ihr Sehen als Fotografen brauchen. Sebastião Salgado beschreibt diesen Zustand so:

„Ich reise neun Monate im Jahr. Der große Vorteil einer Reise ist, dass man mit sich selbst konfrontiert wird. (...) Man findet heraus, was wirklich wichtig ist. Eine andere Sprache, ein anderes Klima, eine andere Umgebung. In diesem Moment besteht die größte Möglichkeit, bei sich selbst zu sein, die Welt zu sehen, die Luft zu riechen, die Orte zu spüren. (...) Wir sind Tiere wie alle anderen auch, nur dass wir unseren Instinkt verloren und ihn getötet haben. Wenn wir reisen, finden wir ihn wieder, indem wir andere beobachten und uns auf den Augenblick einlassen." (Salgado 2000)

Pentti Sammallahti konstatiert: "Bliebe ich zuhause, würde ich blind werden. Ich brauche Distanz, um zu sehen." 10

Sehen und die Gewohnheit - der Maler Avigdor Arikha legt einen tieferen Zusammenhang zwischen ihnen offen. Im alltäglichen Leben, erklärt er, ist unser Blick durch den Vorgang des Wiedererkennens konditioniert.

\begin{abstract}
„Das bedeutet, dass wir im Grunde nur das sehen, was wir wiedererkennen können. Aber um ein Bild oder eine Zeichnung zu schaffen, die vibriert, muss man sich diesem Einfluss entziehen. Nicht wiedererkennen, sondern erkennen und sich in einen ursprünglichen Zustand versetzen. (...) Ein Betrachter (...), dem es nicht gelingt, seine Intentionen, seine Vorurteile und sogar sein Denkvermögen abzulegen, wird nichts sehen. Denn der offene Blick ist der Blick der Sinne.“11
\end{abstract}

Auf Reisen entgehen die Fotografen der Eichung auf Wiedererkennen. In unbekannten Ländern sind ihre Routinen der Wahrnehmung außer Kraft gesetzt. Nichts ist vertraut, weder die Natur, das Klima, die Bauweise der Häuser und Städte, noch die gesellschaftlichen Normen, die üblichen Verhaltsweisen der dort lebenden Menschen, die Abläufe ihres alltäglichen Lebens, ganz zu schweigen von ihrer Sprache. Zuweilen vermgen die Fotografen nicht einmal die Buchstaben der Schrift auf den Schildern zu entziffern. Nichts ist ihnen selbstverständlich. Sie vermgen nicht einzuschätzen, was normal und was ungewhnlich ist. Sie knnen nichts erwarten. Viel stärker als in ihrem gewohnten Umfeld sind sie auf ihre Beobachtungsfähigkeiten angewiesen, auf Präsenz in der gegenwärtigen Situation, auf ein Maximum an Offenheit ihres Geistes und ihrer Sinne. Ein Zustand, der dem Zuhren in Sprachen, die man nur begrenzt beherrscht, nicht unähnlich ist. In beiden Situationen sind

\footnotetext{
${ }^{10}$ Sammallahti, zitiert auf der Homepage des Finnish Institute of London: http://www . finnish-institute.org.uk/en/articles/649-pentti-sammallahti-here-far-away. bersetzung aus dem Englischen von der Verfasserin.

${ }^{11}$ Gesprächsausschnitt mit Avigdor Arikha im Film von O’Bryne, Raphaël (2001): Henri Cartier-Bresson. L'amour tout court/ Einfach Liebe. Les films Lou, ARTE France. Transkription von der Verfasserin.
} 
wir aufgefordert, das, was uns an Wissen und intuitiver Kenntnis fehlt, durch Offenheit, Konzentration und Beobachtung wettzumachen.

Oft führt mich der Mangel an Kenntnissen und Kompetenz im Arabischen und Tschechischen an den Rand des Scheiterns oder auch mitten hinein: ich bin unbeholfen, verhalte mich daneben, mache mich lächerlich, ich bekomme nur die Hälfte mit, verstehe etwas vllig falsch, ich leide an der ungenügenden Feinabstimmung meiner Reaktion auf die Situation, fühle mich zuweilen schmerzlich reduziert. Aber manchmal reißt dieser offenkundige Mangel an Wissen, das fehlende ,Panzerhemd der Gewohnheiten', ein Fenster auf, durch das etwas von der Welt, vom Leben, von einem anderen Menschen in besonderer Intensität zu mir hereinstrmt. Wie der einzigartige Klang einer menschlichen Stimme.

Vielleicht liegt hier der Grund, warum sich mir Stimmen, die Arabisch und Tschechisch sprechen, in besonderer Weise ins Gedächtnis eingraviert haben.

$* * * * * *$

Auch wenn dies auf den ersten Blick nicht so erscheinen mag, so ist es doch zu großen Teilen mein berufliches Arbeitsfeld, das den Entstehungshintergrund des vorliegenden Essays bildet. Seit einigen Jahren arbeite ich als wissenschaftliche Mitarbeiterin an der Professur Deutsch als Zweitsprache der Universität Regensburg mit dem inhaltlichen Schwerpunkt des Sprachenerwerbs von Kindern und Jugendlichen im Kontext von Migration. Vor kurzem nahm ich an einem Forschungskolloquium teil, auf dem eine Doktorandin von ihrem Dissertationsprojekt berichtete. Anhand von narrativen Interviews mit bilingual aufgewachsenen Personen der Zweiten Einwanderergeneration, die nun Eltern geworden sind, untersucht sie die Erfahrungen und Motive, die diese Väter und Mütter bei der Weitergabe ihrer Sprachen an ihre Kinder leiten. Die junge Wissenschaftlerin stellte ihr methodisches Vorgehen vor sowie ihre bisherigen vorläufigen Ergebnisse. Die erste Wortmeldung im Anschluss an den Vortrag kam von einem Professor des Fachbereichs ,Deutsch als Zweitsprache'. Er fragte die Referentin, wie sie selbst als Angehrige der Zweiten Einwanderergeneration zu dem mglichen Vorwurf der Voreingenommenheit stehe. Die Promovendin entgegnete, ob ebenso WissenschaftlerInnen ohne Migrationshintergrund, die Aspekte der Wirklichkeit von Menschen ohne Migrationshintergrund erforschten, aufgefordert würden, zum mglichen Vorwurf ihrer Voreingenommenheit Stellung zu beziehen. Der Professor ohne Migrationshintergrund schwieg, und es kam zu keiner weiteren Klärung der Thematik.

Ich schildere diesen kurzen Wortwechsel, weil er wesentliche Ausgangspunkte des vorangestellten Essays in sich bündelt. Die Frage des Zuhrers birgt Wahrnehmungsmuster, die meines Erachtens im Fachbereich ,Deutsch als Zweitsprache' bis heute nachhaltige Wirkung zeigen. Ein Wahrnehmungsmuster, das sich in der Frage und insbesondere in der scharfsinnigen Replik der Doktorandin offenbart, könnte man als ,majoritäre Erfahrungsblindheit' 
bezeichnen. ,Majoritäre Erfahrungsblindheit' bedeutet, dass Angehörige einer Majorität ${ }^{12}$ das eigene Denken, Empfinden und Tun als das Allgemeine, das Universelle erachten, während sie dasjenige von Angehörigen einer Minorität als etwas Spezifisches wahrnehmen, das besonderen Prägungen unterliegt. ,Majoritäre Erfahrungsblindheit' verweist auf den blinden Fleck in der Wahrnehmung, der einen übersehen lässt, dass man als Angehörige/ $r$ einer Majorität selbst ebenfalls den spezifischen Einflüssen des eigenen Lebenskontextes ausgesetzt war und ist, die es gerade als WissenschaftlerIn in der eigenen Forschung - so weit es möglich ist - zu reflektieren und transparent zu machen gilt. Hierauf werde ich noch zu sprechen kommen. Ein weiteres Wahrnehmungsmuster, das der Frage des Zuhörers zugrunde liegt und meines Erachtens mit dem Phänomen der ,majoritären Erfahrungsblindheit' zusammenhängt, ist die in den Humanwissenschaften verbreitete skeptische Haltung gegenüber Erfahrungen. Biographischer Nähe, persönlicher Erfahrung von WissenschaftlerInnen im Forschungsfeld wird häufig mit Misstrauen begegnet. In der Vertrautheit mit der untersuchten Lebenswelt sieht man die Gefahr von Voreingenommenheit lauern, während biographische Ferne zum Forschungsobjekt als Garant von Neutralität erscheint, als Gewährleistung von größtmöglicher Objektivität und Offenheit - wesentlichen Voraussetzungen der wissenschaftlichen Wahrheitssuche. Während das Risiko der Blickverzerrung aufgrund persönlicher Nähe des Öfteren Thema im wissenschaftlichen Diskurs ist, wird die Frage, zu welchen Arten der Blickverzerrung Unvertrautheit und große Distanz zum untersuchten Wirklichkeitsbereich führen können, weitaus seltener reflektiert.

Dieses Nachwort ist nicht der geeignete Ort, um die Hintergründe der wissenschaftlichen ,Erfahrungsskepsis' näher zu betrachten. Ich werde nur einige Spotlichter auf ihre Symptome im Fachbereich ,Deutsch als Zweitsprache' werfen, die einen entscheidenden Anstoß zum Schreiben des Essays bildeten.

Stellt man sich die wissenschaftliche Erkenntnissuche bildhaft in Metaphern der Optik vor, dann würde man im Fachbereich ,Deutsch als Zweitsprache' sehr viele, scharf geschliffene Linsen sehen, die auf den Wirklichkeitsbereich der Schule gerichtet sind und damit einhergehend auf die schulleistungsbezogenen Aspekte der Sprachlichkeit von SchülerInnen. Die Frage, wie mehrsprachig aufwachsende Kinder und Jugendliche aus migrierten Familien möglichst rasch und effizient umfassende zielsprachliche Kompetenzen im Deutschen auf bildungssprachlichem Niveau erlangen, steht im zentralen Fokus. Aspekte ihrer sprachlichen Wirklichkeit, die nicht unmittelbar für die Optimierung ihres Zweitspracherwerbs der deutschen Schulsprache ,verwertbar' erscheinen, haben es hingegen schwer, längerfristige Aufmerksamkeit im Forschungsdiskurs zu erhalten, geschweige denn Niederschlag in bildungspolitischen Rahmenrichtlinien für die Schule zu finden. Das, was an den Rändern oder ganz außerhalb des Fokus vom Fachgebiet ,Deutsch als Zweitsprache' steht, ist beträchtlich. Ich möchte hier nur zwei besonders schwerwiegende Bereiche ansprechen.

Mit der Blickfokussierung auf Aspekte des Zweitspracherwerbs im Kon- 
text Schule geht einher, dass die Sprachlichkeit von Menschen in erster Linie in hierarchischen Kategorien der Kompetenz wahrgenommen und beurteilt wird, wie dies beispielsweise der Gemeinsame Europäische Referenzrahmen für Sprachen widerspiegelt. Darüber hinausgehende Dimensionen, insbesondere Dimensionen, wie Menschen ihre Sprachlichkeit wahrnehmen und erleben, bleiben außen vor. So wissen wir bislang kaum etwas darüber, wie SchülerInnen ihr schulisches Lernen in der Zweitsprache erleben, was es beispielsweise für die schwächeren LeserInnen unter ihnen heißt, wenn ihnen die Sprache in bildungssprachlich verfassten Texten wie eine ,große Mauer' (Wong Fillmore) vorkommt. Auch wie Menschen etwa den Klang von Sprachen und von Stimmen wahrnehmen oder wie ihrer Beobachtung nach ihre Sprachen ihre Verarbeitung von Eindrücken und Erlebnissen prägen, wie sie ihre Erinnerungen und ihr Vorstellungsvermgen formen, wie sie ihre Beziehungen zu anderen Menschen und ihren Selbstbezug beeinflussen, wird selten Beachtung geschenkt. Befunde aus der Mehrsprachigkeitsforschung zur komplexen Rolle, die Sprachen im Innenleben und im zwischenmenschlichen Leben mehrsprachiger Menschen spielen ${ }^{13}$ - in Wechselwirkung mit ihren Lernund Lebenskontexten sowie ihren unterschiedlichen Kompetenzformen in den jeweiligen Sprachen -, finden noch viel zu wenig Widerhall im fachlichen Diskurs von ,Deutsch als Zweitsprache'.

Zusammenhängend mit den Erlebensdimensionen der Sprachlichkeit bleibt ein weiterer grundlegender Bereich aus dem ,Deutsch als Zweitsprache'-Fachdiskurs bislang nahezu ausgespart. Er betrifft die sprachliche Wirklichkeit der SchülerInnen jenseits der Schulmauern, insbesondere ihren primären sprachlichen Lern- und Lebenskontext der Familie. Bis heute gibt es im deutschsprachigen Raum kaum Untersuchungen zu den spezifischen Bedingungen, mit denen Eltern konfrontiert sind, wenn sie ihren Kindern ihre Sprache vermitteln mchten, die im Land einen Minderheitenstatus hat, d.h. als ,Familienangelegenheit' gilt und für den schulischen Bildungsweg als irrelevant erachtet wird. Ebenso wenig werden die Konsequenzen dieser unter erschwerten Umständen stattfindenden $g$ enerationalen $S$ prachweitergabe $\mathrm{f}$ ür Eltern und Kinder erforscht. Bislang kommen die wichtigen Forschungsbefunde $\mathrm{zu}$ den Folgen einer beeinträchtigen gemeinsamen Eltern-Kind-Sprache ${ }^{14}$ bzw. zur Bedeutung einer sprachlich unproblematischen Kommunikation zwischen

\footnotetext{
${ }^{14}$ Aufgrund der spezifischen Bedingungen der generationalen Sprachweitergabe einer Minderheitensprache kommt es häufig z u e iner s prachlichen D iskrepanz $\mathrm{z}$ wischen d en einzelsprachigen Fähigkeiten der Eltern und denen der Kinder - insbesondere wenn die Kinder in dem Land aufwachsen, in das ihre Eltern erst im Erwachsenenalter einwanderten. Sobald die Kinder in Kindergarten und Schule in Kontakt mit der prestigereicheren Landessprache kommen, ,wachsen' ihre Kompetenzen in der Zweitsprache meist ungleich rascher als die ihrer Eltern, während sich die Entwicklung ihrer erstsprachlichen Fähigkeiten (in der Minderheitensprache) oft verlangsamt oder gar stagniert. Das Forschungsgebiet der Heritage-LanguageAcquisition untersucht v.a. die linguistischen Charakteristika dieser Spracherwerbsform bei den Kindern von MinoritätssprachensprecherInnen. Welche einschneidenden Folgen dies für die Kommunikation zwischen Eltern und Kindern haben kann, hat insbesondere Lily Wong Fillmore in einigen ihrer Beiträge (1991, 2000, 2005) in großer Anschaulichkeit zur Sprache gebracht.
} 
Eltern und Kind für das innerfamiliäre Beziehungsgefüge, für die psychische Entwicklung der Kinder, für ihre Resilienz, für ihren Zweitspracherwerb, für ihr schulisches Lernen in erster Linie aus Untersuchungen im nordamerikanischen Raum.

Bereits vor über 30 Jahren schrieben Donald Hansen und Lily Wong Fillmore im Abschlussbericht zu einer Forschungsstudie:

If our goal is to understand second language learning, however, we must look beyond the classroom to the other loci of language learning. And even if our goal is more modest - to understand the role of the classroom in second language acquisition - we must take a perspective that moves beyond the classroom and into the situations, relationships and interchanges that make up the day-to-day lives of second language learners. For what the learner does in the classroom is influenced by perspectives, commitments and understandings that were both learned and supported by other contexts. (Hansen \& Wong Fillmore 1983: 3f)

Leider hat sich diese Weitsicht auf die sprachliche Wirklichkeit mehrsprachig aufwachsender SchülerInnen im Fachbereich ,Deutsch als Zweitsprache' bis heute noch nicht durchsetzen knnen.

Ich bin davon überzeugt, dass ein entscheidender Schritt hin zu einem genaueren Verständnis der sprachlichen Lebensrealität von Menschen in Migrationsgesellschaften darin besteht, ihren Erfahrungen sorgfältigere Beachtung zu schenken und zu erkennen, dass in der Erfahrung von Menschen nicht nur das Erkenntnisrisiko der Voreingenommenheit, sondern ein großes wissenschaftliches Erkenntnispotential liegt. Der sprachlichen Erfahrung von Menschen im Fachbereich ,Deutsch als Zweitsprache' genauere Beachtung zu schenken, erfordert eine verstärkte Öffnung in mindestens vier Richtungen:

\section{Ermutigung von Wissenschaftlerlnnen mit Innenperspektive}

„Der Mensch sieht, selbst wenn er unfehlbar wäre, im anderen nur jenen Teil, für den seine Blickkraft und Blickart reicht.“, schrieb Franz Kafka ${ }^{16}$. Jede Perspektive ist begrenzt und hat ihre spezifischen Unschärfe-, aber auch ihre Schärferelationen. Zum tief greifenden Verständnis eines komplexen Phänomens wie der Sprachlichkeit des Menschen sind verschiedene Blickwinkel zum untersuchten Wirklichkeitsbereich unverzichtbar. Bislang forschen und arbeiten im Fachbereich ,Deutsch als Zweitsprache' überwiegend Menschen ohne familiäre Migrationserfahrung und ohne die Erfahrung, die gesamte Schulbildung in einer Zweitsprache meistern zu müssen. Es gibt also ein bergewicht am,Von-außen-Blick' und einen Mangel an Perspektiven, die die sprachliche Realität von SprecherInnen einer Minoritätssprache und von ZweitsprachenlernerInnen aus eigener Erfahrung kennen. WissenschaftlerInnen mit dieser Innenperspektive gilt es, besonders zu ermutigen und ihnen die Türen zur Forschung zu öffnen. 


\section{Erforschung von Spracherfahrungen}

Um die sprachliche Lebensrealität von Menschen in Migrationsgesellschaften genauer zu verstehen, ist es unabdingbar, dass der Erforschung ihrer Spracherfahrungen in einer kontextsensiblen Weise - d.h. die Wechselwirkungen zwischen den konkreten gegebenen Bedingungen in ihren Lern- und Lebenskontexten und ihrer individuellen Erfahrungsverarbeitung berücksichtigend und analysierend - eine hhere Bedeutung beigemessen wird. Dies erfordert auch, dass man sich mit den hierfür geeigneten methodischen Untersuchungsinstrumenten ${ }^{17}$ vertraut macht und diese entsprechend der jeweiligen Forschungsfragen weiterentwickelt und verfeinert.

\section{Bedeutung von Spracherfahrungen in der LehrerInnenbildung}

Im Kontrast zu den SchülerInnen hat der überwiegende Teil der LehramtStudierenden und der bereits praktizierenden LehrerInnen, die gegenwärtig im Fachbereich ,Deutsch als Zweitsprache' der Universität Regensburg ausund weitergebildet werden, keine familiäre Migrationserfahrung. Eine zentrale Herausforderung der LehrerInnenbildung sehe ich darin, LehrerInnen einen tieferen Einblick in die Erfahrungswelt von mehrsprachig aufwachsenden Kindern aus migrierten Familien zu vermitteln, d.h. in sprachliche Erfahrungen, Lernherausforderungen und -bedürfnisse, die sie aus eigener Anschauung nicht kennen. Einerseits erfordert dies natürlich die Auseinandersetzung mit Erkenntnissen des aktuellen Forschungsstandes zu dem komplexen Geflecht von Einflussfaktoren auf das sprachliche und schulische Lernen der Kinder. Jedoch bleibt dies ein sehr abstraktes Wissen, das der Vorstellungskraft nur schwer den Sprung in die andere Erfahrungswelt der SchülerInnen ermglicht. Eine große Chance für die Lehre sehe ich darin, die wissenschaftlichen Befunde mit sprachlichen Erfahrungsberichten aus erster Hand (schriftlichen Selbstzeugnissen und Interviews) in Bezug zu setzen und gleichzeitig die KursteilnehmerInnen anzuregen, über ihre eigenen Spracherfahrungen nachzudenken. ${ }^{18}$

\footnotetext{
${ }^{17}$ Siehe als berblick in die bestehenden forschungsmethodischen Verfahren: Busch 2013: $32-39$

${ }^{18}$ Viel versprechend ist in dieser Hinsicht das neue sterreichische Rahmenmodell für die LehrerInnenbildung ,Basiskompetenzen Sprachliche Bildung für alle Lehrenden. Deutsch als Unterrichtssprache - Deutsch als Zweitsprache - alle mitgebrachten und schulisch erlernten (Bildungs-) Sprachen - Sprache/n in den Sachfächern'. (http://oesz . at/OESZNEU/UPLOAD/ Basiskompetenzen_sprachliche_Bildung_FINAL.pdf).
} 


\section{Selbstreflexion der Wissenschaftlerlnnen über ihre eigenen Spracherfahrungen}

Die Selbstreflexivität von WissenschaftlerInnen über die sie prägenden Erfahrungen und Sichtweisen hinsichtlich ihres Untersuchungsfeldes wird seit langem als Gütekriterium qualitativer Forschung eingefordert. ${ }^{19}$ Die Untersuchung der Spracherfahrungen anderer Menschen sollte immer einhergehen mit der Introspektion der ForscherInnen hinsichtlich ihrer eigenen Spracherfahrungen und dem Nachdenken über jene Schärfe- und Unschärferelationen, die ihre eigene Perspektive mit sich bringt. ${ }^{20}$ Warum ist die Selbstreflexivität von WissenschaftlerInnen so bedeutsam?

In jedem Verstehen von Welt, schrieb Heidegger, ist Existenz mitverstanden. Es gibt kein neutrales, erfahrungsloses Verstehen. Um zu verhindern, dass das Verstehen eines anderen Menschen „in fruchtloser Reproduktion eigener Vormeinungen und Vorverständnisse endet, muss eben jenes Vorverständnis bewusst gemacht (...) werden“ (Danzer 1995: 213), um seine mögliche Einflussnahme im Prozess der wissenschaftlichen Untersuchung mit bedenken und so weit wie möglich einschränken zu können. Da es sich bei unserem Erfahrungswissen jedoch in großen Teilen um implizites Wissen ${ }^{22}$ handelt, das uns selten in allen Facetten bewusst und verbalisierbar zur Verfügung steht, sind unserer Selbstverständigung Grenzen gesetzt. Niemals wird es möglich sein, die eigenen Prägungen und Motivationen in ihrer Gesamtheit ins Bewusstsein zu holen. Dennoch ist das Bemühen darum unentbehrlich, um das Risiko der eigenen Blickverengung zu verringern, aber auch im Hinblick auf einen weiteren, im Diskurs um die Gütekriterien wissenschaftlicher Forschung seltener beachteten Aspekt der Selbstreflexivität, auf den Donald A. Hansen und Lily Wong Fillmore in ihrer bereits zitierten Forschungsstudie hinweisen:

Our judgements are less harsh, and often more accurate, when we are the ones who lack the language of our social and cultural surrounds. (...) In making our choices to learn or not learn, and in attempting to understand those choices, we tend to exercise broader and more complex perspective than those we use to explain the failure of immigrant children to use English. We tend to look more closely at our own individual habits, tastes and capabilities, and we try to locate those individual characteristics, and our moment-to-moment decisions, in larger social contexts (...). (Hansen \& Wong Fillmore 1983: 5f)

Unser Blick auf uns selbst ist oft kontextsensibler und komplexitätsbewusster, als wenn wir das Tun und Knnen Anderer beurteilen. Wenn wir uns über uns selbst klar zu werden suchen, sind wir hellhriger für die vielfältigen Einflussfaktoren und die unterschiedlichen Dimensionen des Kontextes,

\footnotetext{
${ }^{19}$ Vgl. hierzu u.a. Ines Steinke 2000: 330f.

${ }^{20}$ Als wegweisend für die diesbezügliche Selbstreflexion v on SprachwissenschaftlerInnen und -didaktikerInnen mchte ich auf den von Christine Pearson Casanave und Sandra R. Schecter 1997 herausgegebenen Band hinweisen sowie auf Sandra Kouritzin 2000, auf Aneta Pavlenko 2003 und auf Werner Koller 2002, .2003
} 
die uns beeinfluss(t)en. Zwar gleicht unser Erfahrungswissen keiner sauber geordneten Enzyklopädie, die sich bei Bedarf aus dem Regal unseres Gedächtnisses ziehen und ablesen lässt, dafür macht es sich des Öfteren in Form eines ,Sträubens' in unserem Inneren bemerkbar, wenn uns Sichtweisen und Erklärungen verkürzt oder verzerrt, der von uns erfahrenen Realität nicht gerecht werdend vorkommen. Als ob unsere Erfahrung feine Widerhaken ausfahren würde, die sich gegen das Übersehen von wesentlichen Dimensionen erfahrener Wirklichkeit verwehren. Diese sich aufstellenden Erfahrungswiderhaken sind meines Erachtens ein wichtiges Erkenntnisinstrument, da sie uns veranlassen, hinter die gegebenen Begriffe und Erklärungsmuster zu sehen, tiefer zu gehen, genauer zu beobachten, unsere Linsen feiner zu schleifen - und dies nicht nur, wenn es uns selbst betrifft, sondern auch, wenn wir die Spracherfahrungen anderer Menschen zu begreifen suchen.

Soweit zum Entstehungshintergrund des vorliegenden Essays, in dem ich das letzte Wort dem Literaturwissenschaftler Edward W. Said geben möchte, dem es in nur drei Sätzen gelang, den Kern dessen auszudrücken, was ich in den letzten Seiten in Worte zu fassen versuchte.

Mit all meiner Arbeit versuchte ich immer aufzuzeigen, dass ein sehr facettenreiches und komplexes Erleben der gemeinsame Nenner menschlichen Lebens ist und nicht abstrakte, in der Regel erfundene Vorstellungen von den Unterschieden zwischen ,uns' und ,denen'. (...) Als Akademiker und Intellektuelle haben wir die Aufgabe, die einseitigen Schemata und die abstrakte Art des Denkens, die uns von der konkreten menschlichen Geschichte und Erfahrung in die Gefilde ideologischer Erfindung, metaphysischer Konfrontation und kollektiver Leidenschaft führt, aufzubrechen und abzubauen. Das soll nicht heißen, dass wir nicht über Probleme wie Unrecht und Leid reden können, sondern dass wir dies immer innerhalb eines Kontextes tun müssen, der (...) in Geschichte, Kultur und sozio-ökonomische Realität eingebettet ist. (Said 2004: 75f)

\section{Bibliographie}

[1] Bakri, Mohammad (2006): من يوم ما رحت (Since You Left (Film)). Produced by Avi Kleinberger \& Mohammad Bakri

[2] Busch, Brigitta (2015): "Without language, everything is chaos and confusion." Bodily and emotional language experience and the linguistic repertoire. In: Lüdtke, Ulrike M. (Hrsg.): . Amsterdam: John Benjamins, 273-288

[3] Busch, Brigitta (2013): Mehrsprachigkeit. Wien: Facultas/UTB

[4] Busch, Brigitta (2010): Die Macht präbabylonischer Phantasien. Ressourcenorientiertes sprachbiographisches Arbeiten. In: LiLi - Zeitschrift für Literaturwissenschaft und Linguistik 160, 58-82 
[5] Busch, Brigitta (2006): Language Biographies - approaches to multilingualism in education and linguistic research. In: Busch, Brigitta; Jardine, Aziza; Tjoutuku, Angelika (Hrsg.): . Cape Town: PREAESA Occasional Papers 24, 5-17

[6] Casanave, Christine P. \& Schecter, Sandra R. (Hrsg.) (1997): Mahwah, NJ: Lawrence Erlbaum Associates

[7] Collins, Brian A.; Toppelberg Claudio O.; Suárez-Orozco Carola; O'Connor, Erin \& Nieto-Castañon, Alfonso (2011): Cross-sectional associations of Spanish and English Competence and Well-being in Latino Children of Immigrants in Kindergarten. In: International Journal of the Sociology of Language 208, 5-24

[8] Danzer, Gerhard (1995): Psychosomatische Medizin. Konzepte und Modelle. Frankfurt am Main: Fischer

[9] Hansen, Donald A. \& Wong Fillmore, Lily (1983): Final Report. California University Berkeley. http://files.eric.ed.gov/fulltext/ ED255067.pdf [zuletzt aufgerufen [2016.06.25

[10] Heidegger, Martin (1953/1927): Sein und Zeit. Tübingen: Niemeyer

[11] Kafka, Franz (1920): Aphorismen. Er - Aufzeichnungen aus dem Jahre 1920 (Kapitel 3). Projekt Gutenberg: http://gutenberg. spiegel.de/buch/franz-kafka-aphorismen-166/3 [zuletzt aufgerufen $[2016.06 .25$

[12] Koller, Werner (2003): Mensch und Text in der Sprachfremde: overt und covert. In: Zeitschrift für Interkulturellen Fremdsprachenunterricht 8/2-3, $1-9$

[13] Koller, Werner (2002): Sprachleben - Lebenssprachen. Ein Versuch, die eigene Sprachgeschichte aufzuarbeiten. In: Häcki-Buhofer, Annelies (Hrsg.): Spracherwerb und Lebensalter. Tübingen: Francke, 327-344

[14] Koudelka, Josef (2002): Josef Koudelka. Praha: Torst

[15] Koudelka, Josef (2013): Interview auf dem ,Look3 photography festival' in Charlottesville, Virginia am .2013.6.15 Transkription: http://pdnpulse.pdnonline.com/2013/06/look3-josef-koudelkaon-the-measure-of-a-photographer-courage-and-controllingyour-own-destiny.html [zuletzt aufgerufen [2016.06.25

[16] Kouritzin, Sandra (2000): A Mother's Tongue. In TESOL Quarterly 34/2, 311- 324

[17] Kunze, Reiner (2003): Wo wir zu Hause das Salz haben. Nachdichtungen. Frankfurt am Main: Fischer 
[18] Pavlenko, Aneta (2007): Autobiographic Narratives as Data in Applied Linguistics. In: Applied Linguistics 28/2, 163-188

[19] Pavlenko, Aneta (2006): Bilingual Selves. In: Pavlenko Aneta (Hrsg.): . Clevedon, UK: Multilingual Matters, 1-33

[20] Pavlenko, Aneta (2003): The Privilege of Writing as an Immigrant Woman. In: Casanave, Christine P. \& Vandrick, Stephanie (Hrsg.): . Mahwah, NJ: Lawrence Erlbaum Associates, 211-230

[21] Pöppel, Ernst \& Bao, Yan (2011): Three Modes of Knowledge as Basis for Intercultural Cognition and Communication: A Theoretical Perspective. In: Han, Shihui. \& Pöppel, Ernst. (Hrsg.): Heidelberg: Springer, 215-231

[22] Pöppel, Ernst (2007): Weisen des Wissens. In: Bodensteiner, Paula; Pöppel, Ernst \& Wagner, Ernst (Hrsg): Wissensgenese an Schulen. Beiträge zu einer Bilddidaktik. München: Hanns-Seidel-Stiftung e.V., 11-18

[23] Rumbaut, Ruben G. (2005): Children of Immigrants and Their Achievement: The Roles of Family, Acculturation, Social Class, Gender, Ethnicity, and School Contexts. In: Taylor, Ronald D. (Hrsg.): Greenwich, IT: Information Age Publishing, Inc., 23-59

[24] Rumbaut, Ruben G. (1994): The Crucible Within: Ethnic Identity, SelfEsteem, and Segmented Assimilation among Children of Immigrants. In: International Migration Review 28/4, 748-794

[25] Said, Edward W. (2004): Huntington nicht das Feld überlassen. In: AlMaaly, Khalid (Hrsg.): Die arabische Welt. Zwischen Tradition und Moderne. Heidelberg: Palmyra, 73-81

[26] Salgado, Sebastião (2000): Das Gedächtnis der Welt. Gespräch zwischen Sebastião Salgado und Constantin von Barloewen. In: Lettre International 50 (Herbst 2000). https://www. lettre. de/content/constantinvon-barloewen_das-ged\%c3\%a4chtnis-der-welt [zuletzt aufgerufen [2016.07.05

[27] Samir (2002): Forget Bagdad إنْسَ بغداد שכח מבגדד - jews and arabs - the iraqi connection. (Film) Dschoint Ventschr Filmproduktion

[28] Steinke, Ines (2000): Gütekriterien qualitativer Forschung. In: Flick, Uwe/ Kardorff, Ernst von/ Steinke, Ines. (Hrsg) (2000): Qualitative Forschung. Ein Handbuch. Reinbek bei Hamburg: Rowohlt, 319-331

[29] Svĕrák, Jan (2007): Vratné láhve. (Film) Biograf Jan Svĕrák a Portobello Pictures

[30] Toppelberg, Claudio O. \& Collins, Brian A. (2010): Language, Culture, and Adaption in Immigrant Children. In: 19/4, 697-717

[31] Wong Fillmore, Lily (2005): The Effects of Educational Policies and Practices on Home Languages and Learning. In: Lopez. Antonia (Hrsg.): National Council of La Raza. Washington, DC, 29-40

[32] Wong Fillmore, Lily (2000): Loss of family languages: Should educators be concerned? In: 39, 203-210

[33] Wong Fillmore, Lily (1991): When learning a second language means losing the first. In: 6, 323-346 